\title{
Keratitis Caused by Onchocerciasis: Wolbachia Bacteria Play a Key Role
}

\author{
G. Kluxen ${ }^{1}$ and A. Hoerauf ${ }^{2}$ \\ ${ }^{1}$ Augenärztliche Überörtliche Gemeinschaftspraxis und Praxisklinik, Wermelskirchen, \\ 2Institute of Medical Microbiology, Immunlology and Parasitology (IMMIP), \\ University Clinic of Bonn, Bonn,
}

Germany

\section{Introduction}

Human beings are the only known important reservoir of Onchocerca volvulus which causes onchocerciasis. The adult worms are usually found in subcutaneous nodules and have an average longevity of approximately 15 years. The parasitic worm releases millions of offspring (microfilariae) which migrate through the skin and can enter the anterior or posterior regions of the eye. While alive, the microfilariae appear to cause little or no inflammation, even being in the anterior chamber. However, when they die, either by natural attrition or after chemotherapy, the host response to degenerating worms can result in ocular inflammation (keratitis, uveitis and optic neuritis) that causes progressive loss of vision. Blindness therefore tends to occur in adulthood after many years of infection.

\section{Ocular onchocerciasis}

The affection starts with a slight conjunctival injection. In Africans an enhancement of the pigmentation at the limbus has also been observed. When phlyctenule-like lesions occasionally appear at the limbus, microfilariae are found in this alteration as well. However, they do not contribute to the development of the keratitis. Here they are only found in a convolution of inflamed cells.

It seems clear that the microfilariae enter the cornea from the limbus via Schlemm's canal and trabeculae (Maertens,1981). The conditions described above, like a slight conjunctival injection, an enhancement of the pigmentation and occasional phlyctenule-like lesions at the limbus indicate the movement of microfilariae in and out of the eye through the conjunctiva.

\subsection{Punctate subepithelial stromal keratopathy}

The corneal disease typically develops as successive showers of subepithelial lesions on both eyes. The epithelium itself is usually normal and does not stain with either bengal rose or fluorescein. The fluffy opacities („,snow flakes“, Kératite ponctuée, Keratitis punctata) lie in the superficial layers of the stroma in the interpalpebral region near the limbus (Fig.1) and are accompanied by a considerable amount of photophobia and lacrimation and sometimes by a temporary but slight impairment of visual acuity. They are due to an oedema developing around the dead microfilariae. They may remain for a longer time but 
eventually resolve without trace, but these lesions tend to develop more and more as long as intracorneal death of the worms continues; and when the keratopathy progress moves on lesions in the deeper stroma occur.

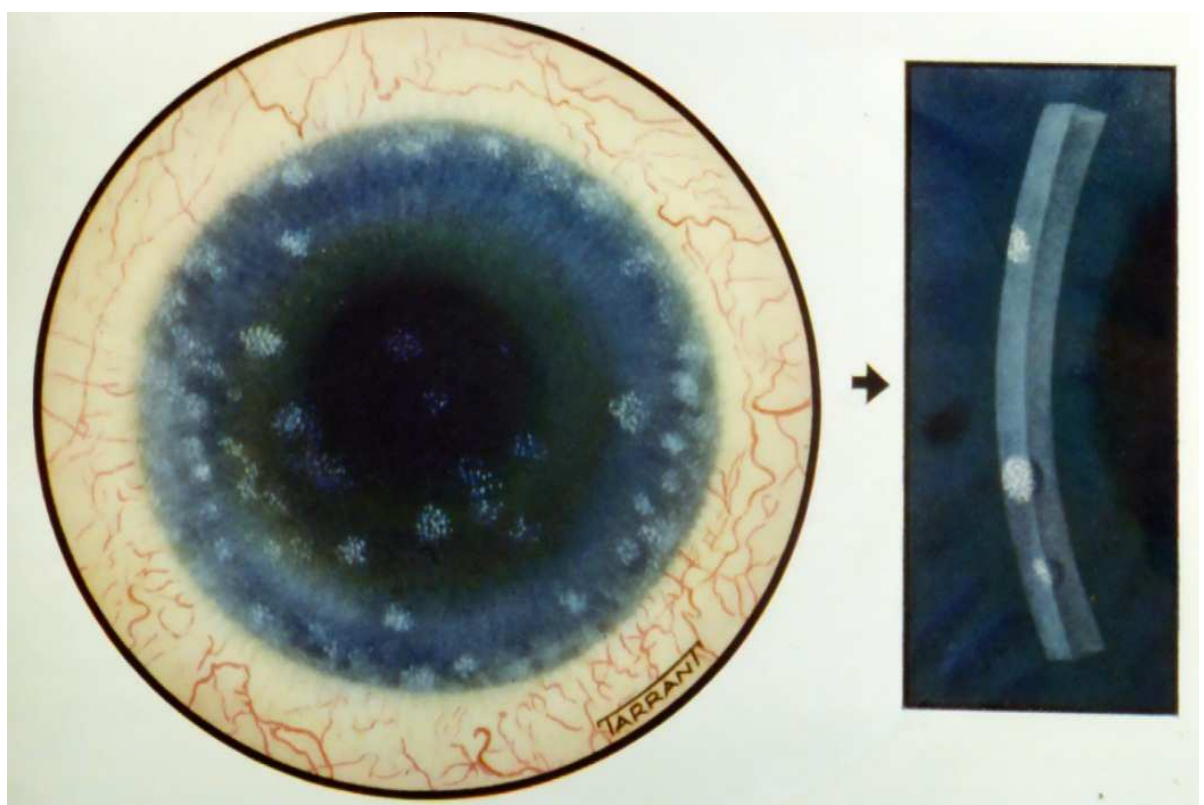

Fig. 1. Well-marked onchocercal keratitis as punctate subepithelial stromal keratopathy in a European, focal section of the cornea showing the punctate infiltration of the anterior subepithelial layers (Choyce, 1958).

Following the terminology of Duke-Elder and Leigh (Duke-Elder, 1965; Duke-Elder \& Leigh, 1966) this keratitis is termed: Punctate subepithelial stromal keratopathy (Fig.1). The opacities of subepithelial facet formation appear also as immune response after perforating graft keratoplasty (Pleyer, 1997). An increasingly evident subepithelial keratopathy caused by onchocerciasis with a variation in infiltration size is also called linear keratitis (Keratitis linearis, Kératitis linéaire) and demonstrates the characteristic appearance of "cracked ice" (glace brisée) (Woodruff et al., 1963). The ephithelium of the cornea remains even and transparent for a long time.

Microfilariae in the cornea are predominantly positioned in a horizontal angle along the corneal nerves (Maertens,1981). They remain in this one position for a long time and then move laboriously through the interstitium (stroma) (Fig.2). After the administration of diethylcarbamazine (DEC) microfilariae tend to move into the cornea. They squirm and roll, then die and elongate stretched. At this point a punctate subepithelial stromal keratopathy (keratitis punctate, punctate keratitis) develops (WHO,1982). The dead microfilariae become opaque, decay and then disappear. Over the next few weeks and months the generated opacity of the cornea can either improve, remain as it is or worsen. Histologically there are eosinophil and neutrophil granulocytes around the dead microfilariae in the cornea stroma. The latter ones are attracted by Wolbachia (Saint André et al., 2002; Gentil \& Pearlman, 2009). 


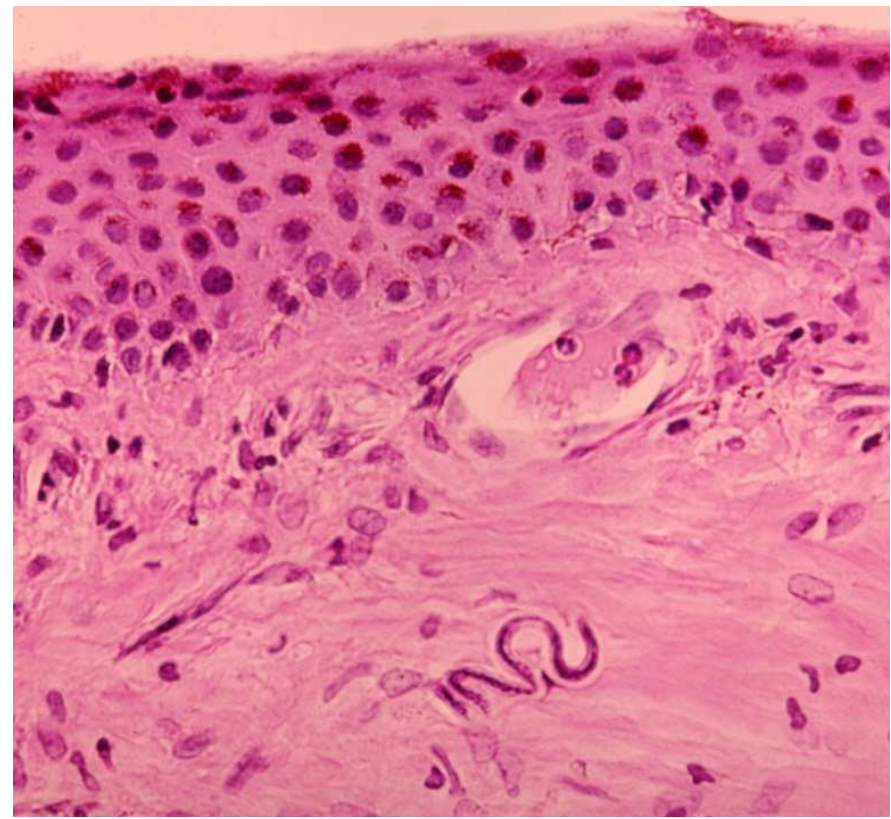

Fig. 2. A microfilaria of Onchocerca volvulus in a corneal scar of the stroma, H.E. staining, by courtesy of Hj.Trojan

In Central America cases in this particular phase were already called interstitial keratitis (Branly,1956), while the European authors in Africa used to call a keratopathy only interstitial if minor vascularisation was also present. However, the cornea affected by ocular onchocerciasis partially remains transparent for a long time (Fig.3, Fig.4).

\subsection{Band-shaped keratopathy and sclerosing keratitis}

The grey opacity is always being separated from the limbus by a clear zone. The limbal edge is sharp while the axial edge slowly fades away, tailing off in scattered spots and cracks. As a general rule of the band-shaped keratopathy the opacity spreads slowly over some years towards the center until the two segments from temporal and nasal meet. However, when a sclerosing keratitis occurs in onchocerciasis it usually evolves from a confluence of several foci of invasion around the lower half of the limbus (Fig.3), typically from tongues between the 3 and 9 o'clock positions, and progresses as an apron round the lower half of the cornea. The upper part with the center of the cornea is at this time initially clear (Fig.4). A few superficial blood vessels, lying at first between the epithelium and Bowman's membrane, invade the cornea. The progress gradually spreads upwards; the invading vessels break through Bowman's membrane and often penetrate into the stroma. In an acute destructive process the keratocytes as well as the fibrillae suffer from necrosis, and in a reparative stage keratocytes multiply and various degenerative changes occur with the development of fibrosis in the stroma, where eosinophils as well are components of the cellular infiltrates, and changes in the overlying epithelium which typically becomes heavily pigmented by the migration of pigmented cells in and from the limbus. 


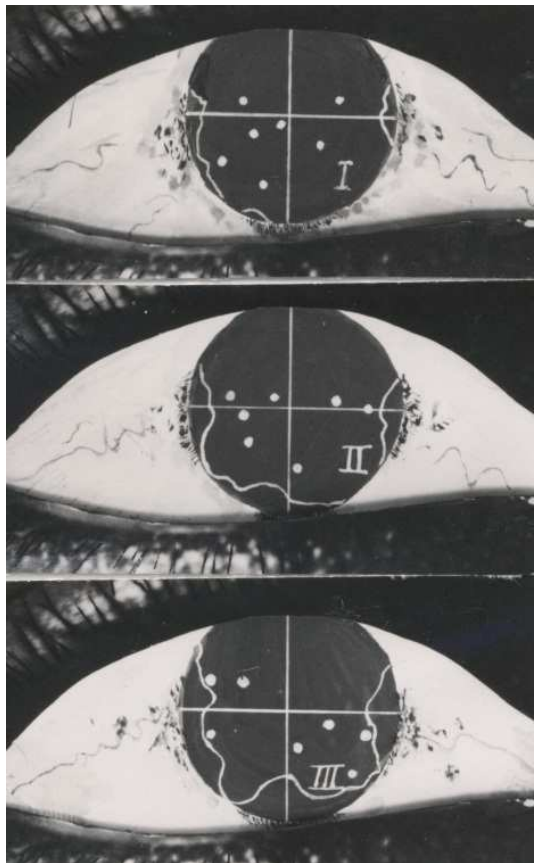

Fig. 3. Development in progress of the sclerosing keratopathy over two years in a young African man with ocular onchocerciasis. The boarder of corneal infiltration from the limbus is shown by the line; the points are larger opacities from punctate subepithelial stromal keratopathy.

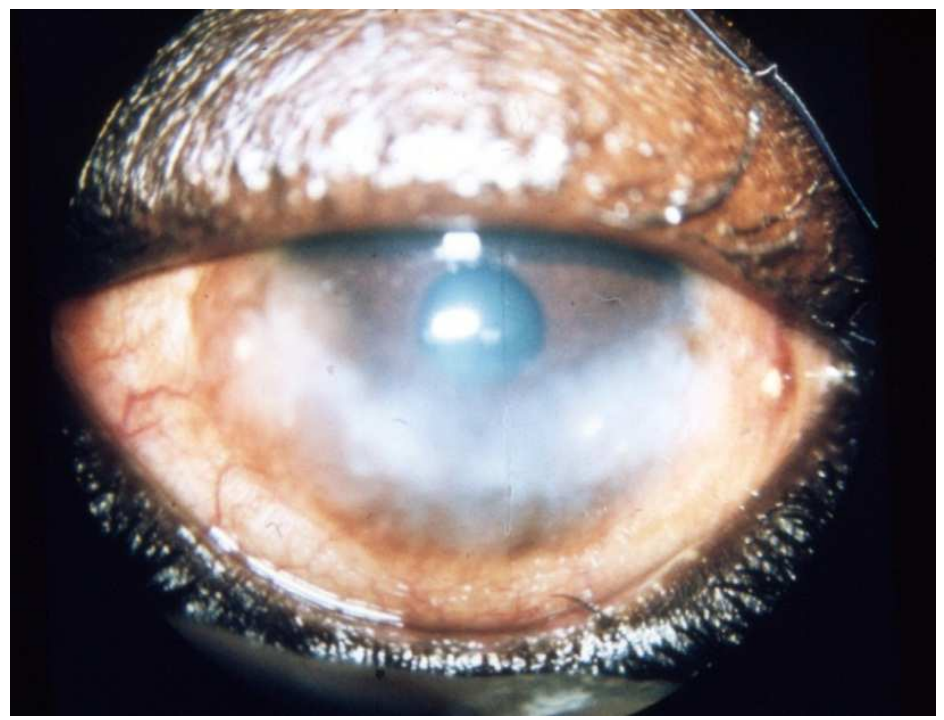

Fig. 4. Keratitis semilunaris, a form of the band-shaped keratopathy, by courtesy of Hj.Trojan 
The cornea has thickened by now, has an uneven surface and is no longer transparent (Fig.5, Fig.6). There are callosities (scars, adherences, synechia) in the inside of the eye and a hypotension can foster the development of a phthisis bulbi, a hypertension a staphyloma of the cornea (Fig.6). The affected eye has turned blind irrevocably and usually the process repeats itself almost simultaneously in the other eye. The cases of sclerosing keratitis increase drastically in endemic onchocerciasis regions particularly in affected people above the age of sixty (Rodger \& Maertens 1977).

\subsection{Discussion of the clinical picture (with differential diagnosis)}

\subsubsection{Keratitis through onchocerciasis is not an infectious but an inflammatory condition}

In ocular onchocerciasis cases the subepithelial opacities in the peripheral cornea initially occur in almost even large patches. They are not a specific pathognomonic indication but are rather an epiphenomenon in a series of infectious eye diseases; they are similar to the facet formations caused by adenovirus- or chlamydia-keratitides (Bialasiewicz \& Jahn,1989). However, these keratitides each have their small individual characteristics. They also occur in zoster and varicella with characteristically larger patches, and were further observed in leprosy and avitaminosis (Maertens,1981). The latter diseases are predominantly inflammatory responses, just like the inflammatory reaction after keratoplasty (Preyer, 1997).

A sclerosing keratitis or band-shaped keratopathy is a late complication occurring in all chronic uveitides therefore this condition of the ocular onchocerciasis is not a specific pathognomonic indication. When a sclerosing keratopathy is developing (Fig.5, Fig.6), other major processes adherent to an uveitis have already occurred in the eye since this severe syndrome cannot develop isolated in the cornea. A keratitis caused by onchocerciasis may not be termed infectious, but it is an infestation like ophthalmomyiasis or cysticercosis (Kanski et al., 2005) and is mainly an inflammatory condition.

\subsubsection{Subepithelial facet formations occur also in Dimmer's nummular keratitis}

Dimmer's nummular keratitis is a slowly-developing benign keratitis without an accompanying conjunctivitis and usually unilaterally, characterized by disc-shaped facet infiltrates in the superficial layers of the corneal stroma. It occurs endogenous in small areas of Central Europe, particularly in Austria, where Friedrich Dimmer [1855-1926] worked (Duke-Elder \& Leigh,1965). Sporadic reports about similar cases have come from East Europe and America, China and South Africa. The disease appears among young landworkers and the incidence is seasonal, the peak period being after the harvest in the autumn. The most striking feature is that the opacities occur deep in the parenchyma and mainly central in location.

\subsubsection{How pathology develops in onchocerciasis and what is new?}

In the anterior segment, microfilariae infiltrate the cornea, where they die as a result of antifilarial therapy or by natural attrition. It was thought that this strong inflammatory reaction after the death of the microfilariae in the eye was probably due to a release of toxins. This is not the fact, but research has identified a major cause (Saint André et al., 2002): Wolbachia endosymbionts. 


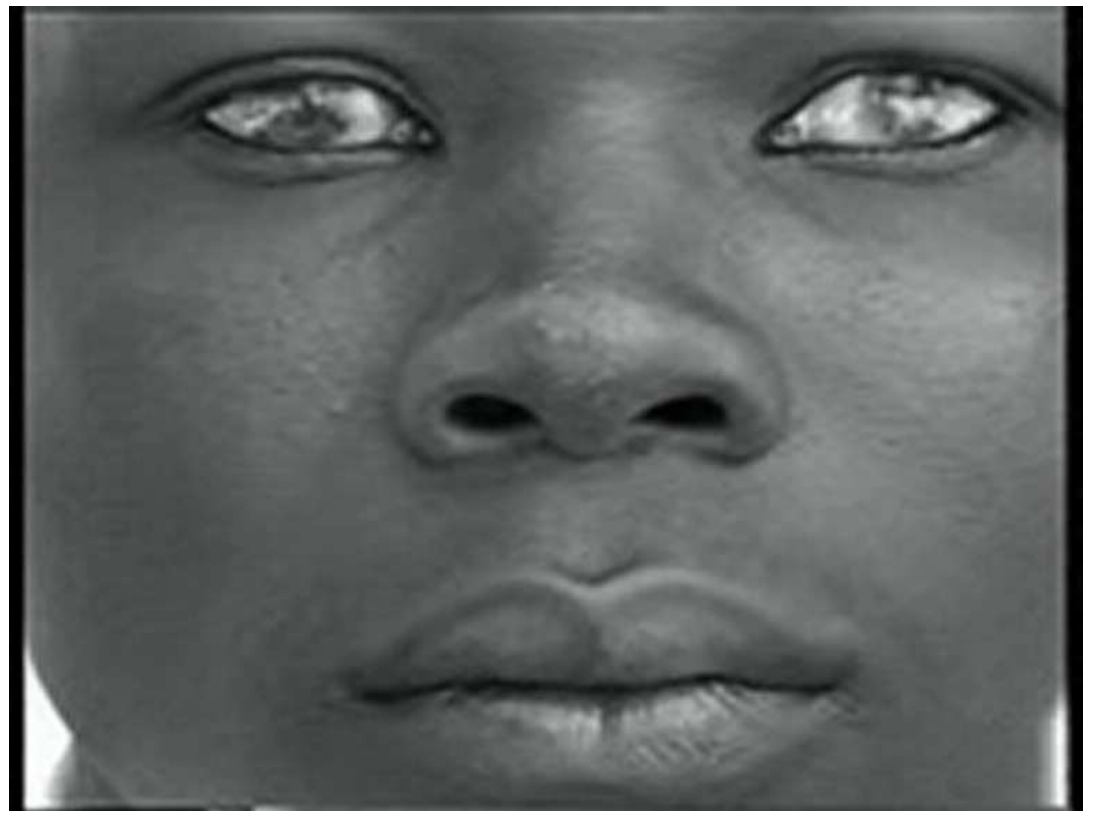

Fig. 5. General view of the corneal opacity, here band-shaped sclerosing keratopathy in a horizontal axis, the young man from Bossangoa is blind with amaurosis.

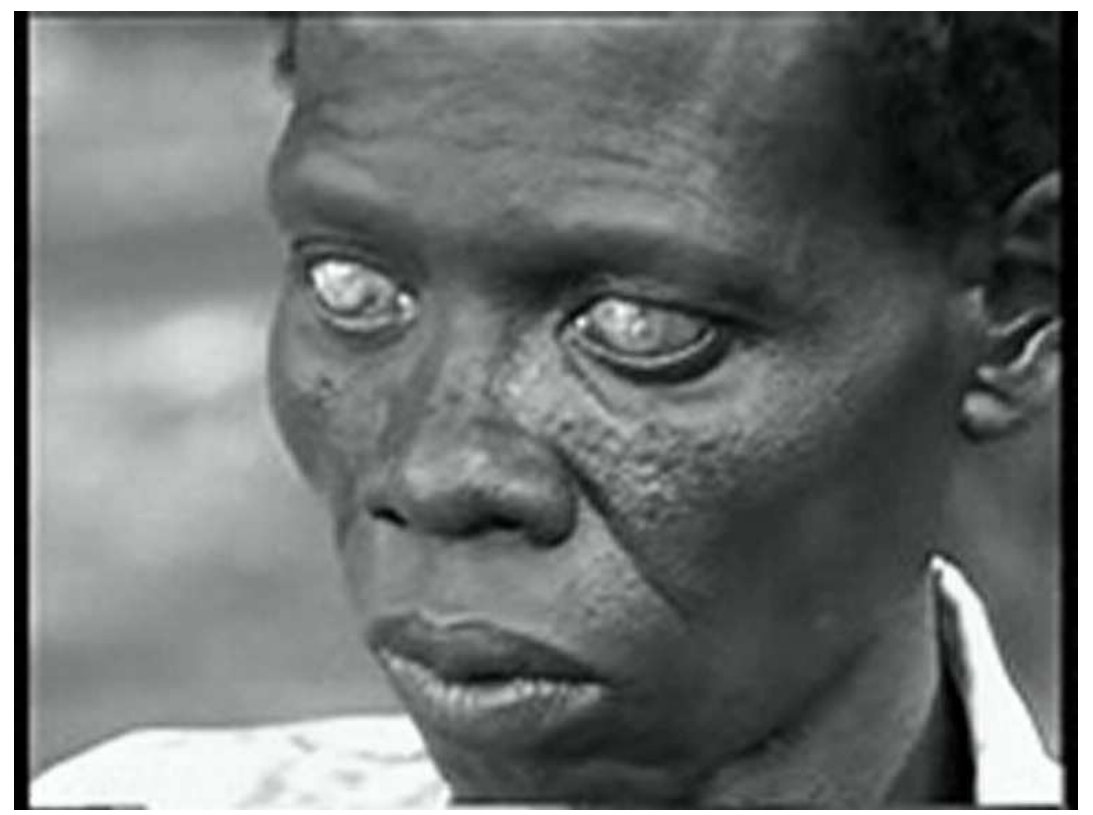

Fig. 6. General view of the corneal opacity, sclerosing keratitis and staphylomata in both sides, amaurosis, man from Bossangoa 


\section{Prevalence of keratitis caused by onchocerciasis}

Knowledge on prevalence and incidence of keratitis through onchocerciasis is limited, and currently there are no surveys available considering the prevalence and incidence of keratitis caused by onchocerciasis in Bossangoa in the North-West of the Central African Republic. In 1994, when the mass-distribution of ivermectin in the area had started, a survey on blindness and visual impairment had been undertaken in this district (Schwartz et al., 1997). The region, with a population of around 100,000, was particularly hyper- and mesoendemicly infected with onchocerciasis. Blindness and visual impairment caused by onchocerciasis was found in 219 cases (bilateral blindness 98, unilateral blindness 49 and visual impairment 72) of 6086 inhabitants. This demonstrates a prevalence of Onchocercaattributable ocular pathology including punctate keratitis of $3.6 \%$. The major causes for blindness in the region (prevalence of $2.2 \%$ ) were found to be onchocerciasis $(73.1 \%)$, cataract $(16.4 \%)$, trachoma $(4.5 \%)$, and glaucoma $(2.2 \%)$. Continuous monitoring of the Bossangoa district was interrupted, because the eye clinic, as well as most of Bossangoa's other buildings, was destroyed in 2002 during the revolution. The eye clinic has been reconstructed since and ophthalmic work is being continued in the town, but it would still be too dangerous to undertake a visit to the district before peace is restored in the area. It remains a conflict area and CDTI (Community directed treatment with Ivermectin) guidelines are never met. A few years after ivermectin therapy had been introduced a decreased number of cases of blindness, mainly attributable to onchocerciasis, had been observed in a comparable community in the North-West sector of the Central African Republic after five years of ivermectin treatment (Kennedy et al., 2002), but there were no changes in the prevalence of main ocular lesions. The mass-distribution of the medication had been interrupted with the outbreak of the conflict. In order to be effective the drug Mectizan ${ }^{\circledR}$ (Ivermectin) must be given to $65 \%$ of all community members for 15-20 years, which correlates with the length of the worm's life cycle.

\section{Wolbachia endosymbionts}

In the worms of Onchocerca volvulus endosymbionts (bacteria called Wolbachia) are located in a specialized anatomical and physiological microenvironment. In adult filarial worms, Wolbachia are mainly found throughout the hypodermal cells of the lateral cords, and are in the embryos and microfilariae. The worm relies on these intracellular living bacteria for its homeostasis. The association between Wolbachia and filarial nematodes is obligatory and not parasitic. It is a mutual partnership (Fenn \& Blaxter, 2007). The hypodermal lateral cord, where Wolbachia are mainly found, may be considered as a special organ for Wolbachia. The first appearance of Wolbachia in the scientific literature was a description of the bacteria within ovaries of mosquitoes (Hertig,1936; Kozek \& Rao, 2007). Onchocerca volvulus and other nematodes harbour endosymbiotic Wolbachia bacteria throughout their life cycles, and they are passed on to the next generation of worms through the oocyte in a vertical transmission like an inherited strain.

As nematode Wolbachia lack factors like genetic selection or recombination, the rapid development of resistance to any drug found to have antiwolbachial activity would be hindered. Data from Wolbachia genomes can be used to better understand the biology of these organisms (Pfarr \& Hoerauf, 2005). It is easy to work out why this bacteria needs to live inside a cell by looking at genome content. For example, Wolbachia of Brugia malayi does 
not have the capacity to synthesise all of the amino acids required for protein synthesis and so must import these from the host cell. On the other hand, the filarial worms themselves are apparently unable to synthesize riboflavins or haem endogenously, but their Wolbachia has a full set of genes to provide these important co-factors (Yamada et al., 2007).

\subsection{Wolbachia play the main role in causing river blindness}

It has become increasingly clear that Wolbachia play a major role in the pathogenesis of ocular onchocerciasis (river blindness) in the human host. Neutrophil infiltration and development of corneal disease occur only when Wolbachia are present (Saint André et al. 2002). Eliminating the endobacteria by doxycycline treatment in infected individuals reduces adverse reactions to current drug therapies and even reduces early stages of pathology (Hoerauf et al., 2000; Hoerauf \& Pfarr, 2007).

The development of the pathology in the infected person is now thought to be a hyperimmune response to worm antigens (Hoerauf \& Pfarr, 2007). But particularly Wolbachia are strong inducers of the immune responses. A reaction from dead worm material (without Wolbachia) into contact with the filarial-infected host is the recruitment of surrounding eosinophils and macrophages, infiltrations in the skin and other tissues.

The most striking feature is that Wolbachia, these "bugs within the bug", are inherent in some filarial worms that infect man and animals, contribute to severe pathological manifestations of filarial infections, and offer a novel approach to chemotherapy and control of filarial infections, especially onchocerciasis.

\subsection{Evidence in favour of the role of Wolbachia in the pathogenesis of onchocerciasis}

\subsubsection{Wolbachia contribute to severe pathological manifestations}

Human monocytes incubated with $O$. volvulus extracts containing Wolbachia stimulated the production of proinflammatory and chemotactic cytokines compared with extracts from $O$. volvulus nodules in the absence of Wolbachia (Brattig et al., 2001). Furthermore, Wolbachia are required for recruitment of neutrophils, innate inflammatory cells, to the onchocercomata (skin nodules containing O. volvulus), as the number of neutrophils in nodules from doxycycline-treated individuals (Fig.7) is greatly reduced compared with untreated patients (Brattig et al., 2001; Saint André et al.; 2002). Neutrophils are only found surrounding worms which contain Wolbachia.

Wolbachia are strong inducers of the immune response. A connection between the very severe filarial pathology in onchocerciasis and Wolbachia infection has also been demonstrated by an in vivo model for blindness in mice (Saint André et al., 2002). The development of neutrophil infiltration and development of a corneal haze in mice after injection of worm extract into the cornea is dependent upon the presence of Wolbachia as $O$. volvulus extract depleted of Wolbachia (Fig.7) does not induce keratitis which is also not induced when these mice do not have a functional Toll-like receptor (TLR) 4 molecule (Hise et al., 2003, Saint André et al., 2002). TLRs are key receptors in the innate immune reaction to foreign antigens. Also TLR 2 plays a role in the development of ocular pathology (Daehnel et al., 2007; Gentil \& Pearlman, 2009). Strong candidates and inducers of the immune response are Wolbachia surface protein and surface glycolipoproteins/glycolipids (Fenn \& Blaxter, 2007). 

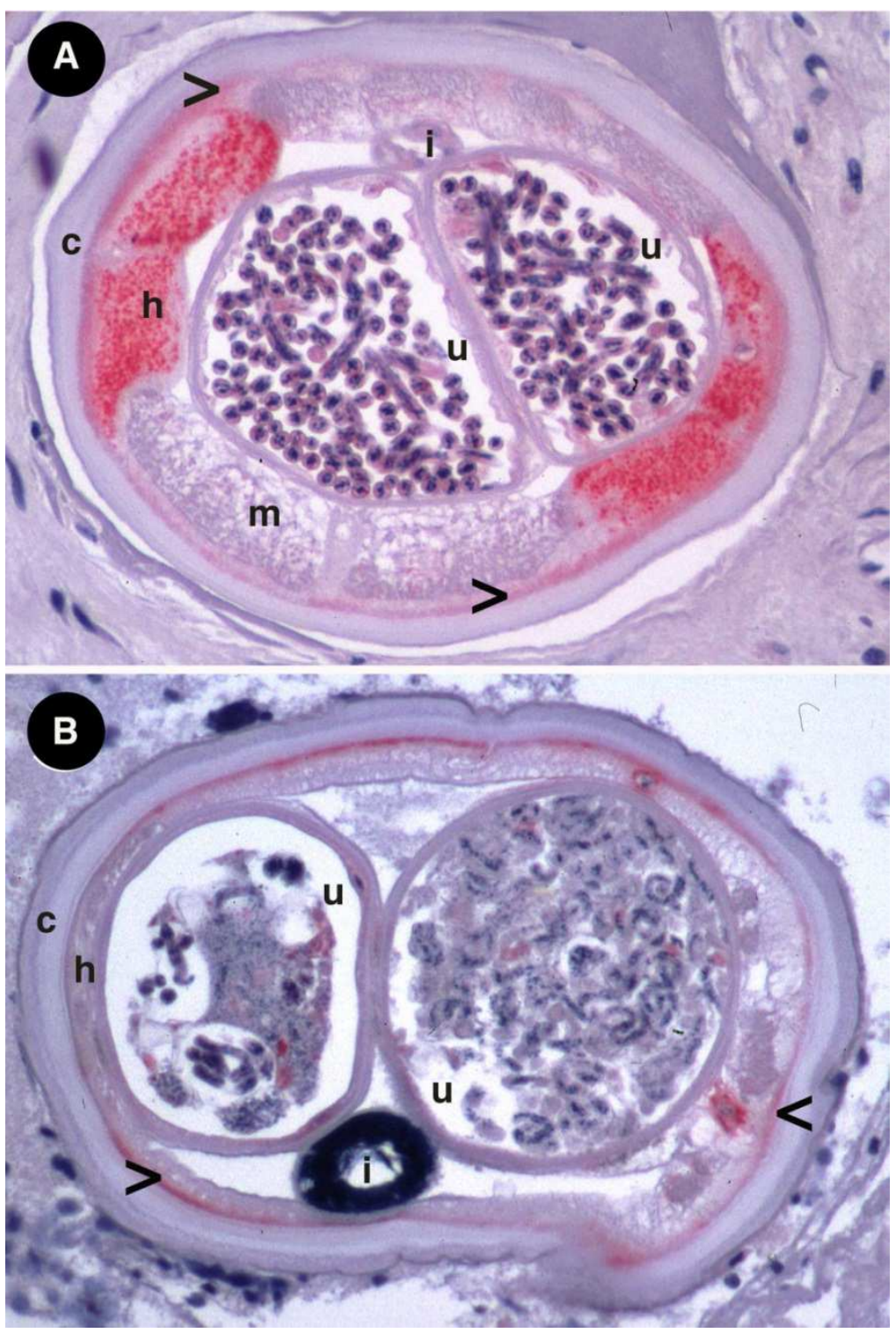

Fig. 7. Immunohistology of midbody cross-sections of female Onchocerca volvulus filariae. Staining with rabbit antiserum against bacterial hsp-60 was used. (A) Worm from control: Bacteria are red stained in the hypodermal cords (h) and in the embryos; cuticle (c), musculature $(\mathrm{m})$, intestine (i), uteri (u) show normal embryogenesis with stretched microfilariae: $x 180$. (B) Worm from patient after doxycycline treatment (ivermectin + doxycycline for 6 weeks, nodulectomy 4 months later): No bacteria are detectable in the hypodermis, a weak staining is shown in areas of mitochondrial density (arrows). Embryos in uteri are degenerated and embryogenesis is interrupted: x 135 (Hoerauf et al., 2000) by courtesy of Elsevier Science. 
A recent finding showed that Wolbachia numbers are more abundant in O. volvulus sampled from infections where severe ocular disease is common, compared to samples from a forested area where blindness is rare (Higazi et al., 2005). In this study, the strain from the savannah shown to cause more severe ocular disease had significantly higher Wolbachia loads compared with a less virulent strain from a forested area.

\subsubsection{Wolbachia contribute to the adverse reactions after microfilaria treatment}

Most of the evidence supporting a role for Wolbachia in the pathogenesis of filarial diseases stems from posttreatment adverse reactions in infected individuals. For example, systemic treatment of onchocerciasis patients with diethylcarbamazine (DEC) causes rapid death of the microfilariae in the skin and eyes, resulting often severe posttreatment side effect the so called "Mazzotti reaction". The severity of the "Mazzotti reaction" is dependent on the number of microfilariae containing Wolbachia in the skin and eyes (Hoerauf \& Pfarr, 2007). Reactions include fever, headache, dizziness, myalgia, arthralgia, tachycardia, ciliary injection, severe pruritus and enlargement of lymph nodes. However, this test is absolutely out-mode now. The reactions resemble the >erisipela de la costa < of Guatemala, which also develops during the needling of head-nodules and the killing of adult worms resulting in a severely thickened face (Kluxen, 2011).

\subsection{An autoimmological reaction in the retina}

Twenty years ago it had been suggested that autoimmunological reactions resulting from cross-reactivity between parasite antigens and components of eye tissues contribute to development of ocular onchocerciasis (Braun et al.,1991). The Onchocerca volvulus antigen Ov39 (39 kDa) is cross-reactive with a retinal antigen of $44 \mathrm{kDa}$ and induces ocular inflammation in rats after immunization (McKechnie et al., 2002). This reaction is thought to develop without a contribution of Wolbachia.

\section{Conclusion}

Findings from studies on infected individuals and animal models demonstrate that endosymbiotic Wolbachia have a profound effect on the pathogenesis of the disease. Pathology of ocular onchocerciasis starts with the induction of cytokines by macrophages and monocytes dependent on the presence of Wolbachia. A potential molecule that is mediating the inflammatory response is Wolbachia surface protein, which elicits a strong inflammatory response via TLR 2 and TLR 4 (Daehnel et al., 2007; Hise et al., 2003; Saint André et al., 2002; Brattig et al., 2004; Gentil \& Pearlman, 2009).

The strain of $O$. volvulus shown to cause more severe ocular disease had significantly higher Wolbachia loads compared with another, less virulent strain, indicating a correlation between virulence and Wolbachia in ocular onchocerciasis (Higazi et al., 2005). One implication may be that Wolbachia boosts immune responsiveness toward filarial antigens, facilitating the clearance of microfilariae and the development of immunopathogenesis (Pfarr et al. 2007). The severity of severe posttreatment side effects has been associated with the microfilarial load of the body before treatment and the antihelmintic drug used to kill the microfilariae (Hoerauf \& Pfarr, 2007). 
It has become increasingly clear that Wolbachia play a major role in the pathogenesis of ocular onchocerciasis (river blindness) in the human host. Neutrophil infiltration and development of corneal disease occur only when Wolbachia are present (Saint André et al., 2002; Gentil \& Pearlman, 2009). However, the presence of Wolbachia in filariae should not be considered as being solely responsible for all the pathological manifestation seen in filarial infections as a weak reaction from dead worm material can also be observed.

Wolbachia are an excellent target for the development of new antifilarial drugs because of their essential role in worm embryogenesis, development and adult survival (Hoerauf et al., 2007). Eliminating the endobacteria (Fig.7) reduces adverse reactions to current drug therapies and even reduces early stages of pathology. Preliminary data indicate (Daehnel et al., 2007) that the elimination of the endobacteria from the worms by doxycycline treatment in infected individuals will also limit the severity of punctate keratitis.

The current mainstay of mass treatment by the World Health Organization's African Programme for Onchocerciasis Control (APOC) is ivermectin, an antiparasitic agent that kills microfilariae and inhibits the growth and proliferation of offspring for several months at a time; it targets mature microfilariae. But the treatment with ivermectin does not cure the disease. Ivermectin + doxycycline cause sterility in adult worms and thereby directly suppress the embryonic development of the worm. The dosage of doxycycline was 100 $\mathrm{mg} /$ day p.o. for six weeks and the dosage of Mectizan ${ }^{\circledR}$ (ivermectin) was 2 times $150 \mu \mathrm{g} / \mathrm{kg}$ during and $4-6$ months after doxycycline treatment (Hoerauf et al., 2001), and another dosage of $200 \mathrm{mg} /$ day doxycycline for 4 - 6 weeks was sufficient to kill over $60 \%$ of the female worms (Hoerauf et al., 2008; Taylor et al., 2010). Studies have shown that the combination of ivermectin and doxycycline significantly enhanced ivermectin-induced suppression of microfilariae, effectively blocking disease transmission for as long as years, possibly irreversibly.

\section{References}

Bialasiewicz, A.A. \& Jahn, G.J. (1989). Chlamydieninfektionen, Enke, Stuttgart

Branly, M.A. (1956). Über die Onchozerkosis (Morbus Robles). Klinische Monatsblätter für Augenheilkunde, Vol.128, No.1, pp. 1-15

Brattig, N.W.; Büttner, D.W. \& Hoerauf, A. (2001): Neutrophil accumulation around Onchocerca worms and chemotaxis of neutrophils are dependent on Wolbachia endobacteria. Microbes and Infection, Vol.3, pp. 439-446

Brattig, N.W.; Bazzocchi, C. \& Kirschning, C.J. et al. (2004). The major surface protein of Wolbachia endosymbionts in filarial nematodes elicits immune responses through TLR2 and TLR4. Journal of Immunology, Vol.173, pp. 437-445

Braun, G.; McKechnie, N.M.; Connor V.; Gilbert, C.E.; Engelbrecht F., Whitworth J.A. \& Taylor D.W. (1991). Immunological crossreactivity between a cloned antigen of Onchocerca volvulus and a component of the retinal pigment epithelium. Journal of Experimental Medicine, Vol.174, (July 1991), pp. 169-177 www.jem.org

Choyce, D.P. (1958). Some observations on the ocular complications of onchocerciasis and their relationship to blindness. Transactions of the Royal Society of Tropical Medicine and Hygiene, Vol.52, pp. 112-121 
Daehnel, K.; Hise, A.G.; Gillette-Ferguson I.; Pearlman, E. (2007). Wolbachia and Onchocerca volvulus: Pathogenesis of river blindness, In: A. Hoerauf \& R.U. Rao (eds.), Wolbachia: A bug's life in another bug, Issues in Infectious Diseases, Vol.5, pp. 133-145, ISBN 978-3-8055-8180-6

Duke-Elder, St. (1965). Diseases of the outer eye. In: Sir St. Duke-Elder (ed.), System of Ophthalmology, Kimpton, London, Vol.VIII/1, pp. 401-425, ISBN 0-85313-218-6

Duke-Elder, S. \& Leigh, A.G. (1966). Diseases of the outer eye. In: Sir St. Duke-Elder (ed.), System of Ophthalmology, Kimpton, London, Vol.VIII/2, pp. 733-751, ISBN 0-85313218-6

Fenn, K. \& Blaxter, M. (2007). Coexist, cooperate and thrive: Wolbachia as long-term symbionts of filarial nematodes, In: A. Hoerauf \& R.U. Rao (eds.), Wolbachia: A bug's life in another bug, Issues in Infectious Diseases, Vol.5, pp. 66-76, ISBN 978-38055-8180-6

Gentil, K. \& Pearlman, E. (2009). Gamma interferon and interleukin-1 receptor 1 regulate neutrophil recruitment to the corneal stroma in a murine model of Onchocerca volvulus keratitis. Infection and Immunity, Vol. 77, No.4 (April 2009), pp. 1606-1612

Hertig, M. (1936). The rickettsia, Wolbachia pipientis (Gen. et Sp. Nov.) and associated inclusions of the mosquito, Culex pipiens. Parasitology, Vol.28, pp. 453-486

Higazi, T.B.; Filiano, A.; Katholi, C.R.; Dadzie, Y.; Remme, J.H. \& Unnasch T.R. (2005). Wolbachia endosymbiont levels in servere and mild strains of Onchocerca volvulus. Molecular and Biochemical Parasitology, Vol.141, No.1 (May 2005), pp. 109-112 http://ini.sagepub.com

Hise, A.G.; Gillette-Ferguson, I. \& Pearlman, E. (2003). Immunopathogenesis of Onchocerca volvulus keratitis (river blindness): a novel role for TLR 4 and endosymbiotic Wolbachia bacteria. Journal of Endotoxin Research, Vol.9, No.6, pp. 390-394 www.sciencedirect.com

Hoerauf, A.; Volkmann, L.; Hamelmann, C.; Adjei, O.; Autenrieth, I.B.; Fleischer, B. \& Büttner, D.W. (2000). Endosymbiotic bacteria in worms as targets for a novel chemotherapy in filariasis. The Lancet, Vol.355, No.9211 (April 2000), pp. 1242-1243

Hoerauf, A.; Mand, S.; Adjei, O.; Fleischer, B. \& Büttner, D.W. (2001). Depletion of Wolbachia endobacteria in Onchocerca volvulus by doxycycline and microfilaridermia after ivermectin treatment. The Lancet, Vol.357, No. 9266 (May 2001), pp. 1415-1416

Hoerauf, A. \& Pfarr, K. (2007). Wolbachia Endosymbionts: An Achilles' heel of filarial nematodes. In: A. Hoerauf \& R.U. Rao (eds.), Wolbachia: A bug's life in another bug. Issues in Infectious Diseases, Vol.5, pp. 31-51, ISBN 978-3-8055-8180-6

Hoerauf, A.; Specht, M.; Büttner, M.; Pfarr, K., Mand, S. et al. (2008). Wolbachia endobacteria depletion by doxycycline as antifilarial therapy has macrofilaridical activity in onchocerciasis: a randomized placebo-controlled study. Medical Microbiology and Immunology, Vol.197, No.3 (September 2008), pp. 295-311

Kanski, J.J.; Milewski, S.A.; Damato, B.E.; Tannar, V. (2005). Diseases of the ocular fundus. Elsevier Mosby, ISBN 0-723433704, Edinburgh

Kennedy, M.H.; Bertocchi, A.D.; Hopkins, A.D.; Meredith, S.E. (2002). The effect of 5 years of annual treatment with ivermectin (Mectizan $\left.{ }^{\circledR}\right)$ on the prevalence and morbidity of 
onchocerciasis in the village of Gami in the Central African Republic. Annals of Tropical Medicine and Parasitology, Vol.96, No.3, pp. 297-307

Kluxen, G. (2011). Dr. Jean Hissette's research expeditions to elucidate river blindness. Kaden, ISBN: 978-3-922777-99-1, Heidelberg

Kozek, W.J. \& Rao, R.U. (2007). The discovery of Wolbachia in arthropods and nematodes - a historical perspective. In: A. Hoerauf \& R.U. Rao (eds.), Wolbachia: A bug's life in another bug. Issues in Infectious Diseases, Vol.5, pp. 1-14, ISBN 978-3-8055-8180-6

Maertens, K. (1981). Les complications oculaires de l'Onchocercose. Annales de la Société Belge de Médecine Tropicale, Vol.61, pp. 199-224

McKechnie, N.M.; Gürr, W.; Yamada, H., Copland, D. \& Braun, G. (2002). Antigen mimicry: Onchocerca volvulus antigen-specific T cells and ocular Inflammation. Investigative Ophthalmology \& Visual Science, Vol.43, No.2 (February 2002), pp. 411-418 www.iovs.org

Pfarr, K. \& Hoerauf, A. (2005). The annotated genome of Wolbachia from the filarial nematode Brugia malayi: What it means for progress in antifilarial medicine. PLoS Medicine, Vol.2, No.4 (April 2005), pp. 0283-0286 www.plosmedicine.org

Pfarr, K.; Foster, J. \& Slatko B. (2007). It takes two: Lessons from the first nematode Wolbachia genome sequence, In: A. Hoerauf \& R.U. Rao (eds.), Wolbachia: A bug's life in another bug. Issues in Infectious Diseases, Vol.5, pp. 52-65, ISBN 978-3-8055-8180-6

Pleyer, U. (1997). Immunreaktion nach perforierender Keratoplastik. Immunbiologie, Prävention und Therapie. Der Ophthalmologe, Vol.94, pp. 933-950

Rodger, F.C. \& Maertens, K. (1977). Ophthalmology. In: F.C. Rodger (ed.), Onchocerciasis in Zaire. A new approach to the problem of river blindness, Pergamon Press, Oxford U.K., chapter 6, pp. 105-130

Saint André, A.v.; Blackwell, N.M.; Hall L.R.; Hoerauf, A. et al. (2002). The role of endosymbiotic Wolbachia bacteria in the pathogenesis of river blindness. Science, Vol.295, (March 2002), pp. 1892-1895 www.sciencemag.org

Schwartz, E.C.; Huss, R.; Hopkins, A.; Dadjim, B.; Madijitoloum, P.; Hénault, C. \& Klauss, V. (1997). Blindness and visual impairment in a region endemic for onchocerciasis in the Central African Republic. British Journal of Ophthalmology, Vol.81, No.7 (July 2002), pp. 443-447

Taylor, M.J.; Hoerauf, A. \& Bockarie, M. (2010). Lymphatic filariasis and onchocerciasis. The Lancet, Vol.376, No. 9747 (October 2010), pp. 1175-1185

WHO expert group (1982). Pathogénie et traitement de l'onchocercose oculaire : Rapport de la huitième réunion du groupe scientifique de travail «filariose » en collaboration avec le programme de prévention de la cécité. Issue of the World Health Organization, WHO/TDR/FIL/SWG (8)/82.3 (unpublished document, contains the collective views of an international group of experts: Maertens, K.; Thylefors, B.; Zéa-Flores, G.; Dadzié, Y.; Duke, B.O.L.; Rolland, A.; Taylor, H.R.; Donnelly, J.J.; Nussenblatt, R. \& Ottesen, E.A.)

Woodruff, A.W.; Barnley, G.R.; Hooland J.T.; Jones D.E.; McCrae A.W.R.; McLaren D.S. (1963). Onchocerciasis and the eye in Western Uganda. Transactions of the Royal Society of Tropical Medicine and Hygiene, Vol.57, (January 1963), pp. 50-63 
Yamada, R.; Brownlie, J.C.; McGraw, E.A. \& O'Neill, S.L. (2007). Insights into Wolbachia biology provided through genomic analysis, In: A. Hoerauf \& R.U. Rao (eds.), Wolbachia: A bug's life in another bug. Issues in Infectious Diseases, Vol.5, pp. 77-89, ISBN 978-3-8055-8180-6 


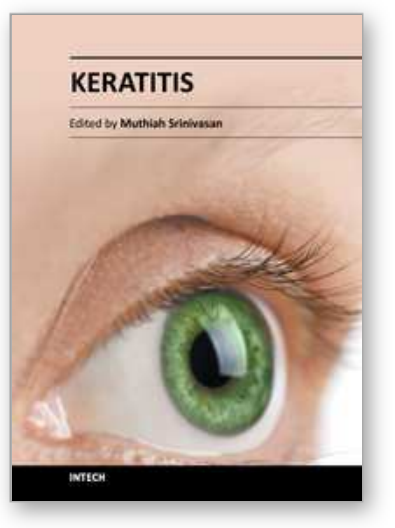

\author{
Keratitis \\ Edited by Dr. Muthiah Srinivasan
}

ISBN 978-953-51-0568-8

Hard cover, 62 pages

Publisher InTech

Published online 25, April, 2012

Published in print edition April, 2012

The 4 chapters in this book focus on investigation, basic and advanced clinical aspects and management of frequently encountered corneal disorders. The authors have covered keratitis theory and practice. Onchocersias, even though found on one continent, has its impact on population, epidemiologists, ophthalmologists, NGOs, public health planners and care providers. The goal of this book is to provide information on ancient eye diseases; their investigation and management to prevent corneal blindness. I acknowledge the great help rendered by Publishing Process Manager and Editor Relations Consultant.

\title{
How to reference
}

In order to correctly reference this scholarly work, feel free to copy and paste the following:

G. Kluxen and A. Hoerauf (2012). Keratitis Caused by Onchocerciasis: Wolbachia Bacteria Play a Key Role, Keratitis, Dr. Muthiah Srinivasan (Ed.), ISBN: 978-953-51-0568-8, InTech, Available from:

http://www.intechopen.com/books/keratitis/keratitis-caused-by-onchocerciasis-wolbachia-bacteria-play-a-keyrole

\section{INTECH}

open science | open minds

\section{InTech Europe}

University Campus STeP Ri

Slavka Krautzeka 83/A

51000 Rijeka, Croatia

Phone: +385 (51) 770447

Fax: +385 (51) 686166

www.intechopen.com

\section{InTech China}

Unit 405, Office Block, Hotel Equatorial Shanghai

No.65, Yan An Road (West), Shanghai, 200040, China

中国上海市延安西路65号上海国际贵都大饭店办公楼405单元

Phone: +86-21-62489820

Fax: +86-21-62489821 
(C) 2012 The Author(s). Licensee IntechOpen. This is an open access article distributed under the terms of the Creative Commons Attribution 3.0 License, which permits unrestricted use, distribution, and reproduction in any medium, provided the original work is properly cited. 